\title{
On Stabilization Problem for Nonlinear Systems with Power Principal Part
}

\author{
M.O. Bebiya and V.I. Korobov \\ Department of Mathematics and Informatics, V.N. Karazin Kharkiv National University \\ 4 Svobody Sq., Kharkiv 61022, Ukraine \\ E-mail: m.bebiya@karazin.ua, \\ vkorobov@univer.kharkov.ua
}

Received June 5, 2015, revised October 21, 2015

In the present paper, the stabilization problem for the uncontrollable with respect to the first approximation nonlinear system with power principal part is solved. A class of stabilizing controls for the nonlinear approximation of this system is constructed by using the Lyapunov function method. It is proved that the same controls solve the stabilization problem for the original nonlinear system. An ellipsoidal approximation of the domain of attraction to the origin is given.

Key words: nonlinear stabilization, nonlinear systems, Lyapunov function method, nonlinear approximation.

Mathematics Subject Classification 2010: 93C10, 93D15, 93D30.

\section{Introduction and Problem Statement}

The stabilization problem for nonlinear systems is an interesting and difficult problem. The most commonly used approach for solving this problem is the method of stabilization with respect to the first approximation [1-4]. The present work is devoted to the stabilization problem for nonlinear systems uncontrollable with respect to the first approximation [5-12].

Consider the system

$$
\left\{\begin{array}{l}
\dot{x}_{1}=u \\
\dot{x}_{i}=\varphi_{i-1}(t, x, u), \quad i=2, \ldots, n,
\end{array}\right.
$$

where $x \in \mathbb{R}^{n}, \mathbb{R}^{n}$ is $n$-dimensional Euclidian space with the norm $\|\cdot\|, u \in \mathbb{R}$ is a control, $\varphi_{i}(t, 0,0)=0$ for all $t \geq 0, i=1, \ldots, n-1$. The functions $\varphi_{i}(t, x, u)$ 
are continuous with respect to all their arguments and are such that in some neighborhood of the origin $\|x\|<\rho$ they can be represented in the form

$$
\varphi_{i}(t, x, u)=c_{i} x_{i}^{2 k_{i}+1}+f_{i}(t, x, u), \quad k_{i}=\frac{p_{i}}{q_{i}}, \quad i=1, \ldots, n-1,
$$

where $p_{i} \geq 0$ are integers, $q_{i}>0$ are odd integers, $c_{i}$ are real numbers such that $\prod_{i=1}^{n-1} c_{i} \neq 0$. The functions $f_{i}(t, x, u)$ are continuous with respect to all their arguments and Lipschitz continuous with respect to $x$ and $u, f_{i}(t, 0,0)=0$ for all $t \geq 0, i=1, \ldots, n-1$.

Assume that there exists $s$ such that $0 \leq s \leq n-2$ and the following condition holds:

$$
k_{i}=0, \quad i=1, \ldots, s \quad \text { and } \quad 0<k_{s+1}<\cdots<k_{n-1} .
$$

We also assume that for some $\alpha_{i}>0$ the functions $f_{i}(t, x, u)$ satisfy the estimates

$$
\left|f_{i}(t, x, u)\right| \leq \alpha_{i}\left(x_{1}^{2 k_{1}+2}+x_{2}^{2 k_{2}+2}+\cdots+x_{n-1}^{2 k_{n-1}+2}\right)
$$

for $\|x\|<\rho, i=1, \ldots, n-1$. For $s=n-2$, condition (3) means that

$$
k_{i}=0, \quad i=1, \ldots, n-2, \quad k_{n-1}=\frac{p_{n-1}}{q_{n-1}}>0 .
$$

For the case $k_{i} \in \mathbb{N}$, for some special class of functions $\varphi_{i}(t, x, u)$, the representation (2) can be obtained by using the Taylor series expansion. As an example, we can take the system

$$
\left\{\begin{array}{l}
\dot{x}_{1}=u \\
\dot{x}_{2}=x_{1}-\sin x_{1} \\
\dot{x}_{3}=x_{2}-x_{2} \cos x_{2}-\sin x_{2}+\frac{1}{2} \sin 2 x_{2},
\end{array}\right.
$$

which is studied below.

The stabilization problem for system (1) is to construct a control $u(t, x)$ such that the equilibrium point $x=0$ of this system is asymptotically stable in the sense of Lyapunov when $u=u(t, x)$.

For the case $k_{i}=0, i=1, \ldots n-2, k_{n-1} \in \mathbb{N}$, the stabilization problem for system (1) was solved in [6]. We present an approach for solving the stabilization problem for nonlinear system (1) in more general case. The approach is based on stabilization of the nonlinear approximation of system (1). For this nonlinear approximation the stabilizing control is constructed in the form $u=u(x)$ and it is proved that this control solves the stabilization problem for the original nonlinear system (1). 
Consider the system

$$
\left\{\begin{array}{l}
x_{1}=u \\
x_{i}=c_{i-1} x_{i-1}^{2 k_{i-1}+1}, \quad i=2, \ldots, n
\end{array}\right.
$$

as a nonlinear approximation of system (1) with right-hand side (2) satisfying conditions (4). This system is uncontrollable with respect to the first approximation.

We will call system (5) with $c_{i}=1, i=1, \ldots, n-1$ the canonical system with power nonlinearities. System (1) is called the system with power principal part if its right-hand side satisfies conditions (4). For the case $k_{i}=0, i=1, \ldots, n-2$, $k_{n-1} \in \mathbb{N}$, the stabilization problem for system (5) was solved in [7].

We note that the terms $c_{i} x_{i}^{2 k_{i}+1}$ are principal for representation (2). We mean that, under conditions (4), system (1) with right-hand side (2) is stabilizable independently of the functions $f_{i}(t, x, u), i=1, \ldots, n-1$. Namely, there exists a control $u=u(x)$, which solves the stabilization problem for system (1), such that for all functions $f_{i}(t, x, u), i=1, \ldots, n-1$ that satisfy estimates (4) there exists a common domain of attraction to the equilibrium point $x=0$.

Similar systems were studied, for example, in [9-12]. The main assumption was that the functions $f_{i}(t, x, u)$ are of bounded growth with respect to $t$, $x_{1}, \ldots, x_{i}, i=1, \ldots, n-1$, and $u$, i.e.,

$$
\left|f_{i}(t, x, u)\right| \leq M\left(\left|x_{i+1}\right|^{p_{i+1}}+\cdots+\left|x_{n}\right|^{p_{n}}\right), \quad i=1, \ldots, n-1
$$

with some additional restrictions imposed on the numbers $p_{i}$. The last condition is an analog of triangularity $[13,14]$. In contrast, conditions (4) mean that the functions $f_{i}(t, x, u)$ are of bounded growth with respect to $t, x_{n}$, and $u$.

The approach for solving the stabilization problem, which was used in [912], is based on a step-wise Lyapunov function and a stabilizing control design. As a result, the Lyapunov function and the stabilizing control were obtained recursively. That is why the practical construction of the stabilizing control is difficult.

In the present work we show that it is possible to construct a Lyapunov function as a quadratic form and find a stabilizing control in an explicit form. The paper is organized as follows. In Sec. 2 the stabilizing control for system (5) with $c_{i}=1, i=1, \ldots, n-1$ is constructed in the form $u=u(x)$. In Sec. 3 it is shown that this control solves the stabilization problem for system (1) with $c_{i}=1, i=1, \ldots, n-1$. Finally, the case $\prod_{i=1}^{n-1} c_{i} \neq 0$ is considered. 


\section{Stabilization of Canonical System with Power Nonlinearities}

In this section we solve the stabilization problem for system (5) for the case $c_{i}=1, i=1, \ldots, n-1$. We begin with considering the nonlinear system:

$$
\left\{\begin{array}{l}
\dot{x}_{1}=u, \\
\dot{x}_{2}=x_{1}^{2 k_{1}+1}, \\
\cdot \cdot \cdot \cdot \\
\dot{x}_{n}=x_{n-1}^{2 k_{n-1}+1}
\end{array} .\right.
$$

where $k_{i}=\frac{p_{i}}{q_{i}}\left(p_{i} \geq 0\right.$ are integers, $q_{i}>0$ are odd integers), $i=1, \ldots, n-1$ satisfy condition (3).

We introduce the following feedback control law:

$$
u(x)=a_{1} x_{1}+a_{2} x_{2}+\cdots+a_{n} x_{n}+\sum_{i=s+1}^{n-1} a_{n-s+i} x_{i}^{2 k_{i}+1},
$$

where $\left\{a_{i}\right\}_{i=1}^{2 n-s-1}$ are negative real numbers, which will be defined below.

Our goal is to provide sufficient conditions under which this control solves the stabilization problem for system (6). To this end, we use the Lyapunov function method. The Lyapunov function $V(x)$ will be found in the form

$$
V(x)=(F x, x),
$$

where $F$ is some positive definite matrix.

We use the following notation:

$$
A=\left(\begin{array}{ccccccc}
a_{1} & a_{2} & \ldots & a_{s} & a_{s+1} & \ldots & a_{n} \\
1 & 0 & \ldots & 0 & 0 & \ldots & 0 \\
\ldots & \ddots & \ddots & \ldots & \ldots & \ldots & \ldots \\
\ldots & \ldots & \ddots & \ddots & \ldots & \ldots & \ldots \\
0 & 0 & 0 & 1 & 0 & \ldots & 0 \\
0 & 0 & 0 & 0 & 0 & \ldots & 0 \\
\ldots & \ldots & \ldots & \ldots & \ldots & \ddots & \ldots \\
0 & 0 & 0 & 0 & 0 & \ldots & 0
\end{array}\right),
$$

$h_{i}=(\underbrace{a_{n-s+i-1}, 0, \ldots, 0,1}_{i}, 0, \ldots, 0)^{T}$ is an $n$-dimensional vector ( 1 is in the $i$-th row), $i=s+2, \ldots, n, A$ is an $(n \times n)$ matrix.

Suppose that the control $u=u(x)$ is applied to system (6). Calculating the derivative of $V(x)$ along the trajectories of the closed-loop system (6), we obtain

$$
\left.\dot{V}(x)\right|_{(6)}=\left(\left(A^{T} F+F A\right) x, x\right)+2 \sum_{i=s+1}^{n-1}\left(F h_{i+1}, x\right) x_{i}^{2 k_{i}+1} .
$$


Note that because the matrix $A$ is singular it is impossible to choose the positive definite matrix $F$ such that the matrix $A^{*} F+F A$ is negative definite. So we try to choose the positive definite matrix $F$ such that the matrix $A^{*} F+F A$ is negative semi-definite. To this end, we consider the Lyapunov matrix equation

$$
A^{T} F+F A=-W,
$$

where $W$ is some given positive semi-definite matrix, the matrix $A$ is given by (9), $F$ is an unknown matrix. We define the matrix $F$ as a positive definite solution of equation (11). Equation (11) is called a singular Lyapunov matrix equation.

In general, matrix equation (11) is not solvable in the class of all positive definite matrices $F$. Therefore we consider equation (11) under the assumption that the matrix $W$ has the form

$$
\left(\begin{array}{cccccc}
w_{11} & \cdots & w_{1 s+1} & w_{1 s+1} \frac{a_{s+2}}{a_{s+1}} & \cdots & w_{1 s+1} \frac{a_{n}}{a_{s+1}} \\
\cdots & \ddots & \cdots & \cdots & \cdots & \cdots \\
w_{1 s+1} & \cdots & w_{s+1 s+1} & w_{s+1 s+1} \frac{a_{s+2}}{a_{s+1}} & \cdots & w_{s+1 s+1} \frac{a_{n}}{a_{s+1}} \\
w_{1 s+1} \frac{a_{s+2}}{a_{s+1}} & \cdots & w_{s+1 s+1} \frac{a_{s+2}}{a_{s+1}} & w_{s+1 s+1} \frac{a_{s+2}^{2}}{a_{s+1}^{2}} & \cdots & w_{s+1 s+1} \frac{a_{s+2} a_{n}}{a_{s+1}^{2}} \\
\cdots & \cdots & \cdots & \cdots & \ddots & \cdots \\
w_{1 s+1} \frac{a_{n}}{a_{s+1}} & \cdots & w_{s+1 s+1} \frac{a_{n}}{a_{s+1}} & w_{s+1 s+1} \frac{a_{s+2} a_{n}}{a_{s+1}^{2}} & \cdots & w_{s+1 s+1} \frac{a_{n}^{2}}{a_{s+1}^{2}}
\end{array}\right) .
$$

In this case, equation (11) has a positive definite solution $F$, but this solution is not unique. In Theorem 1 we describe the class of positive definite solutions of matrix equation (11). First we need the following lemma.

Lemma 1. If the matrix

$$
W_{s+1}=\left(\begin{array}{ccc}
w_{11} & \cdots & w_{1 s+1} \\
\cdots & \ddots & \cdots \\
w_{1 s+1} & \cdots & w_{s+1 s+1}
\end{array}\right)
$$

is positive semi-definite, then the matrix $W$ given by (12) is positive semi-definite.

$\mathrm{P}$ r o o f. To prove that matrix (12) is positive semi-definite, we show that all principal minors of this matrix are non-negative. By $M_{i_{1}, \ldots, i_{k}}^{i_{1}, \ldots, i_{k}}$, we denote the principal minors of the matrix $W$. These minors are formed by rows and columns with the numbers $1 \leq i_{1} \leq \ldots \leq i_{k} \leq n$.

From the positive semi-definiteness of the matrix $W_{s+1}$ we obtain that

$$
M_{i_{1}, \ldots, i_{k}}^{i_{1}, \ldots, i_{k}} \geq 0, \quad 1 \leq i_{1} \leq \cdots \leq i_{k} \leq s+1
$$


Taking into account that the last $(n-s)$ rows and columns of matrix (12) are linearly dependent, from inequality (14) we obtain that

$$
M_{i_{1}, \ldots, i_{k}, i_{k+1}, \ldots, i_{m}}^{i_{1}, \ldots, i_{k}, i_{k+1}, \ldots, i_{m}}=0,1 \leq i_{1} \leq \cdots \leq i_{k}<s+1, s+1 \leq i_{k+1}<\cdots<i_{m} \leq n,
$$

where $m>k+1 \geq 1$, and

$M_{i_{1}, \ldots, i_{k}, i_{k+1}}^{i_{1}, \ldots, i_{k}, i_{k+1}}=\frac{a_{i_{k+1}}^{2}}{a_{s+1}^{2}} M_{i_{1}, \ldots, i_{k}, s+1}^{i_{1}, \ldots, i_{k}, s+1} \geq 0, \quad 1 \leq i_{1}<\cdots<i_{k} \leq s, s+2 \leq i_{k+1} \leq n$,

where $k \geq 0$. Thus the lemma is proved.

Theorem 1. Let the matrices $A$ and $W$ be defined by (9) and (12), respectively. Suppose that the matrix $W_{s+1}$ given by (13) is positive definite, the eigenvalues of the matrix

$$
A_{s+1}=\left(\begin{array}{ccccc}
a_{1} & a_{2} & \cdots & a_{s} & a_{s+1} \\
1 & 0 & \cdots & 0 & 0 \\
\vdots & \ddots & \cdots & \vdots & \vdots \\
\vdots & \vdots & \ddots & \vdots & \vdots \\
0 & 0 & \cdots & 1 & 0
\end{array}\right)
$$

have negative real parts. Define the matrix $F_{s+1}=\left\{f_{i j}\right\}_{i, j=1}^{s+1}$ as a unique positive definite solution of the equation

$$
A_{s+1}^{T} F_{s+1}+F_{s+1} A_{s+1}=-W_{s+1} .
$$

Then the Lyapunov matrix equation (11) is solvable, and the matrix

$$
F=\left(\begin{array}{cccccc}
f_{11} & \cdots & f_{1 s+1} & f_{1 s+1} \frac{a_{s+2}}{a_{s+1}} & \cdots & f_{1 s+1} \frac{a_{n}}{a_{s+1}} \\
\ldots & \ddots & \cdots & \cdots & \cdots & \cdots \\
f_{1 s+1} & \cdots & f_{s+1 s+1} & f_{s+1 s+1} \frac{a_{s+2}}{a_{s+1}} & \cdots & f_{s+1 s+1} \frac{a_{n}}{a_{s+1}} \\
f_{1 s+1} \frac{a_{s+2}}{a_{s+1}} & \cdots & f_{s+1 s+1} \frac{a_{s+2}}{a_{s+1}} & f_{s+2 s+2} & \cdots & f_{s+2 n} \\
\ldots & \cdots & \cdots & \cdots & \ddots & \cdots \\
f_{1 s+1} \frac{a_{n}}{a_{s+1}} & \cdots & f_{s+1 s+1} \frac{a_{n}}{a_{s+1}} & f_{n s+2} & \cdots & f_{n n}
\end{array}\right)
$$

where the elements $\left\{f_{i j}\right\}_{i, j=s+2}^{n}$ are arbitrary real numbers, is a solution of this equation. Moreover, there exist numbers $\left\{f_{i j}\right\}_{i, j=s+2}^{n}$ such that matrix (17) is positive definite.

P r o o f. Due to the assumption that the matrix $A$ has the form (9), the matrix $F=\left\{f_{i j}\right\}_{i, j=1}^{n}$ is a solution of equation (11) if and only if the matrix 
$F_{s+1}=\left\{f_{i j}\right\}_{i, j=1}^{s+1}$ satisfies equation (16) and the elements $f_{i j}, i=1, \ldots, s+1$, $j=s+2, \ldots, n$ satisfy the system

$$
\left\{\begin{array}{l}
f_{1 i} a_{j}+f_{1 j} a_{i}+f_{i+1 j}=-w_{i j}, \\
i=1, \ldots, s, \quad j=s+2, \ldots, n, \\
f_{1 i} a_{j}+f_{1 j} a_{i}=-w_{i j}, \\
i=s+1, \ldots, j, \quad j=s+2, \ldots, n,
\end{array}\right.
$$

where $w_{i j}$ are the entries of the matrix (12).

Note that the last statement is true for equation (11) with an arbitrary symmetric matrix $W=\left\{w_{i j}\right\}_{i, j=1}^{n}$ on its right-hand side. In general, for an arbitrary matrix $A$, equation (11) does not split into the Lyapunov matrix equation (16) and system (18).

Since the eigenvalues of the matrix $A_{s+1}$ have negative real parts, matrix equation (16) has a unique positive definite solution $F_{s+1}$ for an arbitrary positive definite matrix $W_{s+1}$. We recall that this solution can be represented in the form

$$
F_{s+1}=\int_{0}^{\infty} e^{A_{s+1}^{T} t} W_{s+1} e^{A_{s+1} t} d t .
$$

Because the matrix $F_{s+1}=\left\{f_{i j}\right\}_{i, j=1}^{s+1}$ is a solution of equation (16), its elements satisfy the linear system

$$
\left\{\begin{array}{l}
f_{1 i} a_{s+1}+a_{i} f_{1 s+1}+f_{i+1 s+1}=-w_{i s+1}, \quad i=1, \ldots, s+1, \\
2 a_{s+1} f_{1 s+1}=-w_{s+1 s+1} .
\end{array}\right.
$$

From (12) and (17), we have

$$
\begin{array}{r}
w_{i j}=w_{i s+1} \frac{a_{j}}{a_{s+1}}, \quad f_{i j}=f_{i s+1} \frac{a_{j}}{a_{s+1}}, \quad i=1, \ldots, s+1, \quad j=s+2, \ldots, n, \\
w_{i j}=w_{s+1 s+1} \frac{a_{i} a_{j}}{a_{s+1}^{2}}, \quad i=s+2, \ldots, n, \quad j=s+2, \ldots, n .
\end{array}
$$

First we show that the elements of the matrix $F$ defined by (17) satisfy system (18). Using relations (20), we rewrite system (18) in the form

$$
\left\{\begin{array}{l}
f_{1 i} a_{j}+\frac{a_{i} a_{j}}{a_{s+1}} f_{1 s+1}+\frac{a_{j}}{a_{s+1}} f_{i+1 s+1}=-\frac{a_{j}}{a_{s+1}} w_{i s+1} \\
i=1, \ldots, s, j=s+2, \ldots, n \\
2 \frac{a_{i} a_{j}}{a_{s+1}} f_{1 s+1}=-\frac{a_{i} a_{j}}{a_{s+1}^{2}} w_{s+1 s+1} \\
i=s+1, \ldots j, j=s+2, \ldots, n
\end{array}\right.
$$

Multiplying both sides of the first equation by $\frac{a_{s+1}}{a_{j}}$ and both sides of the second equation by $\frac{a_{s+1}^{2}}{a_{i} a_{j}}$, we obtain that the last system is equivalent to system (19). 
Thus the elements of the matrix $F$ defined by (17) satisfy system (18). Therefore the matrix $F$ is the solution of matrix equation (11).

Now we prove that there exist the numbers $f_{i j}, i=s+1, \ldots, n, j=1, \ldots, n$ such that the matrix $F$ defined by (17) is positive definite. Suppose that the elements $\left\{f_{i j}\right\}_{i, j=s+2}^{n}$, for $i \neq j$, are chosen to be arbitrary fixed numbers such that $f_{i j}=f_{j i}$. We denote by $M_{i j}$ the determinant of the $(n-1) \times(n-1)$ matrix that results from deleting row $i$ and column $j$ of the matrix $F$. Then the leading principal minors $\Delta\left(F_{i}\right)$ of the matrix $F$ are defined by

$$
\Delta\left(F_{i}\right)=f_{i i} \Delta\left(F_{i-1}\right)+\sum_{j=1}^{i-1}(-1)^{j+i} f_{j i} M_{j i}, \quad j=s+2, \ldots, n .
$$

We recall that $\Delta\left(F_{s+1}\right)>0$. Now choosing $f_{i i}$ one-by-one so that

$$
f_{i i}>\max \left\{0, \frac{1}{\Delta\left(F_{i-1}\right)} \sum_{j=1}^{i-1}(-1)^{j+i+1} f_{j i} M_{j i}\right\}, \quad i=s+2, \ldots, n,
$$

we obtain that $\Delta\left(F_{i}\right)>0, i=s+2, \ldots, n$. Taking into account that the matrix $F_{s+1}$ is positive definite, we have that $\Delta\left(F_{i}\right)>0, i=1, \ldots, n$ and the matrix $F$ is positive definite. This concludes the proof.

Now we find sufficient conditions under which the control $u=u(x)$ defined by (7) solves the stabilization problem for system (6).

Let the matrix $W_{s+1}=\left\{w_{i j}\right\}_{i, j=1}^{s+1}$ be positive definite. Suppose the matrix $W$ is defined by (12). We define the matrix $F$ as a positive definite solution of the Lyapunov matrix equation (11). Therefore, using Theorem 1, we obtain that (10) takes the form

$$
\left.\dot{V}(x)\right|_{(6)}=-(W x, x)+2 \sum_{i=s+1}^{n-1}\left(F h_{i+1}, x\right) x_{i}^{2 k_{i}+1},
$$

where the matrix $F$ is given by (17).

Let us introduce the following notation $b^{i}=-F h_{i}, i=s+2, \ldots, n$. Then

$$
\begin{aligned}
b_{j}^{i} & =-\left(f_{1 j} a_{n-s+i-1}+f_{j s+1} \frac{a_{i}}{a_{s+1}}\right), \quad j=1, \ldots, s+1, \quad i=s+2, \ldots, n, \\
b_{j}^{i} & =-\left(f_{1 s+1} \frac{a_{j}}{a_{s+1}} a_{n-s+i-1}+f_{j i}\right), \quad j=s+2, \ldots, n, \quad i=s+2, \ldots, n .
\end{aligned}
$$

We chose the numbers $a_{n-s+i-1}, s+2 \leq i \leq n$ and $f_{j i}, s+2<i<j \leq n$ such that

$$
b_{j}^{i}=0, \quad j=i, \ldots, n, \quad i=s+2, \ldots, n .
$$


Solving the last system, we obtain

$$
\begin{gathered}
a_{n-s+i-1}=-\frac{a_{s+1}}{a_{i}} \frac{f_{i i}}{f_{1 s+1}}, \quad i=s+2, \ldots, n, \\
f_{j i}=\frac{a_{j}}{a_{i}} f_{i i}, \quad j=i+1, \ldots, n, \quad i=s+2, \ldots, n-1 .
\end{gathered}
$$

Thus the matrix $\left\{f_{i j}\right\}_{i, j=s+2}^{n}$, which is formed by the last $(n-s-1)$ rows and the columns of the matrix $F$ given by (17), takes the form

$$
\left(\begin{array}{cccc}
f_{s+2 s+2} & f_{s+2 s+2} \frac{a_{s+3}}{a_{s+2}} & \cdots & f_{s+2 s+2} \frac{a_{n}}{a_{s+2}} \\
f_{s+2 s+2} \frac{a_{s+3}}{a_{s+2}} & f_{s+3 s+3} & \cdots & f_{s+3 s+3} \frac{a_{n}}{a_{s+3}} \\
\cdots & \cdots & \ddots & \cdots \\
f_{s+2 s+2} \frac{a_{n}}{a_{s+2}} & f_{s+3 s+3} \frac{a_{n}}{a_{s+3}} & \cdots & f_{n n}
\end{array}\right) .
$$

It is easy to show that under conditions (25) the matrix $F$ given by (17) is positive definite if and only if

$$
f_{i i}>\frac{a_{i}^{2}}{a_{i-1}^{2}} f_{i-1 i-1}, \quad i=s+2, \ldots, n .
$$

From (22), (23), and (24) it follows that

$$
\begin{gathered}
b_{j}^{i}=\frac{a_{s+1}}{a_{i}}\left(\frac{f_{1 j} f_{i i}}{f_{1 s+1}}-f_{j s+1} \frac{a_{i}^{2}}{a_{s+1}^{2}}\right), \quad j=1, \ldots, s+1, \quad i=s+2, \ldots, n, \\
b_{j}^{i}=\frac{a_{j}}{a_{i}}\left(f_{i i}-f_{j j} \frac{a_{i}^{2}}{a_{j}^{2}}\right), \quad j=s+1, \ldots, i-1, \quad i=s+2, \ldots, n .
\end{gathered}
$$

We choose $f_{i i}>0, i=s+2, \ldots, n$ such that condition (26) holds. Therefore, using (28), we obtain

$$
b_{i-1}^{i}=\frac{a_{i-1}}{a_{i}}\left(f_{i i}-f_{i-1 i-1} \frac{a_{i}^{2}}{a_{i-1}^{2}}\right)>0, \quad i=s+2, \ldots, n .
$$

Finally, we obtain that (21) takes the form

$$
\left.\dot{V}(x)\right|_{(6)}=-(W x, x)-2 \sum_{i=s+2}^{n} \sum_{j=1}^{i-1} b_{j}^{i} x_{j} x_{i-1}^{2 k_{i-1}+1}
$$

where $b_{i}^{j}$ are defined by (27) and (28).

We note that $x_{i}^{2 k_{i}+2}>0$ for $x \neq 0$ because $2 k_{i}+2=\frac{2\left(p_{i}+q_{i}\right)}{q_{i}}, i=1, \ldots, n-1$ is a ratio of even and odd numbers. 
Consider the case $n=2$. It follows from (28) and (29) that

$$
\left.\dot{V}(x)\right|_{(6)}=-(W x, x)-2 b_{1}^{2} x_{1}^{2 k_{1}+2}<0 \quad \text { for } \quad\|x\| \neq 0 .
$$

To prove that the last inequality holds, we consider the following two cases. If $x_{1} \neq 0$, then the last inequality is true because $b_{1}^{2}=\frac{a_{1}}{a_{2}}\left(f_{22}-f_{11} \frac{a_{2}^{2}}{a_{1}^{2}}\right)>0$ and the matrix $W$ given by (12) is positive semi-definite. If $x_{1}=0$, then $-(W x, x)=$ $-w_{11} \frac{a_{2}^{2}}{a_{1}^{2}} x_{2}^{2}<0$ for $x_{2} \neq 0$ and the inequality holds.

Let us introduce the following notation $I_{n, n-s}=\operatorname{diag}(1, \ldots, 1, \underbrace{0, \ldots, 0}_{n-s})$ is an $n \times n$ diagonal matrix, $I_{s+1,1}=\operatorname{diag}(1, \ldots, 1,0)$ is an $(s+1) \times(s+1)$ diagonal matrix, $I_{s+1}$ is the identity matrix of dimension $(s+1) \times(s+1)$.

Now we consider the case $n \geq 3$. Since the matrix $W_{s+1}$ is positive semidefinite, then the following estimate holds

$$
\left(W_{s+1} y, y\right) \geq \lambda_{\min }(y, y), \quad y \in \mathbb{R}^{s+1},
$$

where $\lambda_{\min }>0$ is the smallest eigenvalue of the matrix $W_{s+1}$. Using estimate (30), we obtain that

$$
\left(\left(W_{s+1}-\lambda_{\min } I_{s+1,1}\right) x, x\right)=\left(\left(W_{s+1}-\lambda_{\min } I_{s+1}\right) y, y\right)+\lambda_{\min } x_{s+1}^{2} \geq 0,
$$

where $y=\left(x_{1}, \ldots, x_{s+1}\right)^{T}$, i.e., the matrix $W_{s+1}-\lambda_{\min } I_{s+1,1}$ is positive semidefinite.

Let us show that the matrix $W-\lambda_{\min } I_{n, n-s}$ is positive semi-definite. Indeed, the matrix $W_{s+1}-\lambda_{\min } I_{s+1,1}$ is formed by the first $(s+1)$ rows and the columns of the matrix $W-\lambda_{\min } I_{n, n-s}$. Since $W_{s+1}-\lambda_{\min } I_{s+1,1} \geq 0$, using Lemma 1 , we obtain that the matrix $W-\lambda_{\min } I_{n, n-s}$ is positive semi-definite, that is,

$$
\left(\left(W-\lambda_{\min } I_{n, n-s}\right) x, x\right) \geq 0
$$

for all $x \in \mathbb{R}^{n}$.

Let us rewrite equality (29) in the form

$$
\begin{aligned}
\left.\dot{V}(x)\right|_{(6)}=- & \left(\left(W-\lambda_{\min } I_{n, n-s}\right) x, x\right)-\lambda_{\min } \sum_{i=1}^{s} x_{i}^{2}- \\
& -2 \sum_{i=s+1}^{n-1} \sum_{j=1}^{i} b_{j}^{i+1} x_{j} x_{i}^{2 k_{i}+1} .
\end{aligned}
$$

We recall that according to Young's inequality for any $a, b, r>0$ we have

$$
a b \leq \frac{1}{1+r} a^{1+r}+\frac{r}{1+r} b^{1+\frac{1}{r}} .
$$


Below we will show that $\left.\dot{V}(x)\right|_{(6)}$ is negative in some deleted neighborhood of the origin. Let us choose the real numbers $r_{j}>0, j=1, \ldots, n-1$ such that the following conditions hold:

$$
\begin{gathered}
0<r_{j}<2 k_{s+1}, \quad j=1, \ldots, s \\
2 k_{j}+1<r_{j}<2 k_{j+1}+1, \quad j=s+1, \ldots, n-1 .
\end{gathered}
$$

Using inequality (33), we obtain the following estimates:

$$
x_{j} x_{i}^{2 k_{i}+1} \leq\left|x_{j}\right|\left|x_{i}\right|^{2 k_{i}+1-r_{j}}\left|x_{i}\right|^{r_{j}} \leq \frac{1}{2}\left(\left|x_{j}\right|^{2}+\left|x_{i}\right|^{4 k_{i}+2-2 r_{j}}\right)\left|x_{i}\right|^{r_{j}},
$$

where $j=1, \ldots, s, i=s+1, \ldots, n-1$, and

$$
x_{j} x_{i}^{2 k_{i}+1} \leq \frac{1}{1+r_{j}}\left|x_{j}\right|^{r_{j}+1}+\frac{r_{j}}{1+r_{j}}\left|x_{i}\right|^{2 k_{i}+1+\frac{2 k_{i}+1}{r_{j}}},
$$

where $j=1, \ldots, i-1, i=s+1, \ldots, n-1$.

From (32), using (36) and (37), we obtain

$$
\begin{gathered}
\left.\dot{V}(x)\right|_{(6)} \leq- \\
\left.+\left(W-\lambda_{\min } I_{n, n-s}\right) x, x\right)-\lambda_{\min } \sum_{i=1}^{s} x_{i}^{2}-2 \sum_{i=s+1}^{n-1} b_{i}^{i+1} x_{i}^{2 k_{i}+2} \\
+\sum_{i=s+1}^{n-1} \sum_{j=1}^{s}\left|b_{j}^{i+1}\right|\left(\left|x_{j}\right|^{2}\left|x_{i}\right|^{r_{j}}+\left|x_{i}\right|^{4 k_{i}+2-r_{j}}\right) \\
+2 \sum_{i=s+2}^{n-1} \sum_{j=s+1}^{i-1}\left|b_{j}^{i+1}\right|\left(\frac{1}{1+r_{j}}\left|x_{j}\right|^{r_{j}+1}+\frac{r_{j}}{1+r_{j}}\left|x_{i}\right|^{2 k_{i}+1+\frac{2 k_{i}+1}{r_{j}}}\right) .
\end{gathered}
$$

It is easy to show that

$$
\sum_{i=s+2}^{n-1} \sum_{j=s+1}^{i-1} \frac{\left|b_{j}^{i+1}\right|}{1+r_{j}}\left|x_{j}\right|^{r_{j}+1}=\sum_{i=s+1}^{n-2} \sum_{j=i+2}^{n} \frac{\left|b_{i}^{j}\right|}{1+r_{i}}\left|x_{i}\right|^{r_{i}+1} .
$$

Therefore inequality (38) takes the form

$$
\begin{gathered}
\left.\dot{V}(x)\right|_{(6)} \leq-\left(\left(W-\lambda_{\min } I_{n, n-s}\right) x, x\right)-\sum_{i=1}^{s}\left(\lambda_{\min }-\sum_{j=s+1}^{n-1}\left|b_{i}^{j+1}\right|\left|x_{j}\right|^{r_{i}}\right) x_{i}^{2} \\
-2 \sum_{i=s+1}^{n-1}\left(b_{i}^{i+1}-\frac{1}{2} \sum_{j=1}^{s}\left|b_{j}^{i+1}\right|\left|x_{i}\right|^{2 k_{i}-r_{j}}-\sum_{j=i+2}^{n} \frac{\left|b_{i}^{j}\right|}{1+r_{i}}\left|x_{i}\right|^{r_{i}-2 k_{i}-1}\right. \\
\left.-\sum_{j=s+1}^{i-1} \frac{r_{j}}{1+r_{j}}\left|b_{j}^{i+1}\right|\left|x_{i}\right|^{\frac{2 k_{i}+1-r_{j}}{r_{j}}}\right) x_{i}^{2 k_{i}+2} .
\end{gathered}
$$


Using the notation

$$
\beta_{j}=\min _{1 \leq i \leq s}\left\{\left(\frac{\lambda_{\min }}{(n-s-1)\left|b_{i}^{j+1}\right|}\right)^{\frac{1}{r_{i}}}\right\}, \quad j=s+1, \ldots, n-1,
$$

we have

$$
\lambda_{\text {min }}-\sum_{j=s+1}^{n-1}\left|b_{i}^{j+1}\right|\left|x_{j}\right|^{r_{i}}>0, \quad i=1, \ldots, s
$$

for $x_{j}$ such that $\left|x_{j}\right|<\beta_{j}, j=s+1, \ldots, n-1$.

Consider a family of functions $g_{i}: R \longrightarrow R$ of the form

$$
\begin{gathered}
g_{i}(x)=2 b_{i}^{i+1}-\sum_{j=1}^{s}\left|b_{j}^{i+1}\right||x|^{2 k_{i}-r_{j}}-2 \sum_{j=i+2}^{n} \frac{\left|b_{i}^{j}\right|}{1+r_{i}}|x|^{r_{i}-2 k_{i}-1} \\
-2 \sum_{j=s+1}^{i-1} \frac{r_{j}}{1+r_{j}}\left|b_{j}^{i+1}\right||x|^{\frac{2 k_{i}+1-r_{j}}{r_{j}}}, \quad i=s+1, \ldots, n-1 .
\end{gathered}
$$

From (34) and (35) it follows that

$$
\begin{gathered}
2 k_{i}-r_{j}>0, \quad j=1, \ldots, s, \quad i=s+1, \ldots, n-1, \\
r_{i}-2 k_{i}-1>0, \quad \frac{2 k_{i}+1-r_{j}}{r_{j}}>0, \quad j<i, \quad i=s+1, \ldots, n-1 .
\end{gathered}
$$

Therefore the functions $g_{i}(x), i=s+1, \ldots, n-1$ are continuous, symmetric, and $g_{i}(x)$ have their global maximum at the point $x=0$. Moreover,

$$
g_{i}(0)=2 b_{i}^{i+1}>0, \quad i=s+1, \ldots, n-1 .
$$

We denote by $x_{i}^{*}$ the smallest positive root of the equation $g_{i}(x)=0$. Then, using (41), we obtain

$$
g_{i}(x)>0 \quad \text { for } \quad|x| \leq x_{i}^{*}, \quad i=s+1, \ldots, n-1 .
$$

From (39) we obtain

$$
\begin{gathered}
\left.\dot{V}(x)\right|_{(6)} \leq-\left(\left(W-\lambda_{\min } I_{n, n-s}\right) x, x\right)-\sum_{i=1}^{s}\left(\lambda_{\min }-\sum_{j=s+1}^{n-1}\left|b_{i}^{j+1}\right|\left|x_{j}\right|^{r_{i}}\right) x_{i}^{2} \\
-\sum_{i=s+1}^{n-1} g_{i}\left(x_{i}\right) x_{i}^{2 k_{i}+2}<0
\end{gathered}
$$

for $x \in \mathbb{R}^{n}$ such that $\left|x_{i}\right|<\min \left\{\beta_{i}, x_{i}^{*}\right\}, i=s+1, \ldots, n-1$, and $\|x\| \neq 0$. 
Let us show that the last inequality holds. Indeed, consider the following two cases. If $x_{i} \neq 0$ for some $i$ such that $1 \leq i \leq n-1$, then it follows from (31), (40), and (42) that inequality (43) is true. If $x_{1}=x_{2}=\cdots=x_{n-1}=0$, then

$$
-\left(\left(W-\lambda_{\min } I_{n, n-s}\right) x, x\right)=-w_{s+1 s+1} \frac{a_{n}^{2}}{a_{s+1}^{2}} x_{n}^{2}<0 \quad \text { for } \quad x_{n} \neq 0
$$

and inequality (43) is true.

Inequality (43) implies that the control $u=u(x)$ defined by (7) solves the stabilization problem for system (6).

Let us construct an ellipsoidal approximation of the domain of attraction to the equilibrium point $x=0$ for the case $n \geq 3$. To this end, we find the largest $c>0$ such that the ellipsoid $(F x, x) \leq c$ is contained in the set

$$
\Omega=\left\{x \in \mathbb{R}^{n}:\left|x_{i}\right| \leq \gamma_{i}, i=s+1, \ldots, n-1\right\},
$$

where $\gamma_{i}=\min \left\{\beta_{i}, x_{i}^{*}\right\}, i=s+1, \ldots, n-1$.

In this connection we consider the extremal problem of finding a minimum of the function $(F x, x)$ under the restriction $\left(x, e_{i}\right)=\gamma_{i}$, where $i$ is a fixed number such that $s+1 \leq i \leq n-1, e_{i}$ is the $i$-th column of the $n \times n$ identity matrix. Let us introduce the Lagrange function

$$
L(x, \lambda)=(F x, x)-\lambda\left(\left(x, e_{i}\right)-\gamma_{i}\right) .
$$

Let $x^{*}$ be a point of global minimum. The necessary condition of the extremum gives that $L_{x}\left(x^{*}, \lambda\right)=2 F x^{*}-\lambda e_{i}=0$. Thus we have $x^{*}=\frac{1}{2} \lambda F^{-1} e_{i}$. Substituting $x^{*}$ in the restrictions, we get $\frac{1}{2} \lambda\left(F^{-1} e_{i}, e_{i}\right)=\gamma_{i}$. Finding $\lambda$ from the last equation, we have $x^{*}=\frac{\gamma_{i}}{\left(F^{-1} e_{i}, e_{i}\right)} F^{-1} e_{i}$. Finally, we obtain $\left(F x^{*}, x^{*}\right)=\frac{\gamma_{i}^{2}}{\left(F^{-1} e_{i}, e_{i}\right)}$.

So we have proved that, for $n \geq 3$, the domain of attraction to the equilibrium point $x=0$ of the closed-loop system (6) contains the ellipsoid

$$
\Phi=\left\{x \in \mathbb{R}^{n}:(F x, x)<c, \quad c=\min _{s+1 \leq i \leq n-1} \frac{\gamma_{i}^{2}}{\left(F^{-1} e_{i}, e_{i}\right)}\right\} .
$$

Now we can summarize the previous discussion and formulate the main result of this section. In the following theorem we describe the solution of the stabilization problem for system (6).

Theorem 2. Suppose $a_{i}<0, i=1, \ldots, s+1$ are real numbers such that the eigenvalues of the matrix $A_{s+1}$ given by (15) have negative real parts, and $a_{i}<0, i=s+2, \ldots, n$ are arbitrary real numbers. Suppose $W_{s+1}$ given by (13) is an arbitrary positive definite matrix. Let the matrix $F_{s+1}=\left\{f_{i j}\right\}_{i, j=1}^{s+1}$ be a unique positive definite solution of the equation (16). Choose the numbers $f_{i j}$, 
$i=j, \ldots, n, j=s+2, \ldots, n$ such that the conditions (25) and (26) are satisfied. Define the matrix $F$ by (17); and define the numbers $a_{n-s+i-1}, i=s+2, \ldots, n$ by (24). Then the control $u=u(x)$ defined by (7) solves the stabilization problem for system (6). Moreover, the domain of attraction to the equilibrium point $x=0$ of the closed-loop system (6) contains ellipsoid (44) in the case $n \geq 3$ and coincides with the whole space in the case $n=2$.

\section{Stabilization of Systems with Power Principal Part}

In this section we solve the stabilization problem for system (1). First consider the case $c_{i}=1, i=1, \ldots, n-1$. Then system (1) takes the form

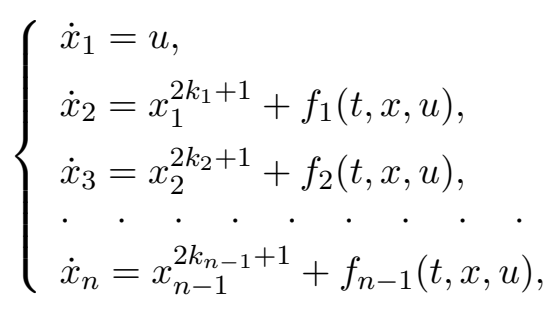

where $k_{i}=\frac{p_{i}}{q_{i}}, p_{i} \geq 0$ are integers, $q_{i}>0$ are odd integers, $i=1, \ldots, n-1$.

As before, we assume that the numbers $k_{i}, i=1, \ldots, n-1$ satisfy condition (3). Moreover, we assume that the functions $f_{i}(t, x, u)$ satisfy conditions (4), i.e., for some $\alpha_{i}>0$ we have

$$
\left|f_{i}(t, x, u)\right| \leq \alpha_{i}\left(x_{1}^{2 k_{1}+2}+x_{2}^{2 k_{2}+2}+\cdots+x_{n-1}^{2 k_{n-1}+2}\right), \quad i=1, \ldots, n-1
$$

when $\|x\|<\rho, \rho>0$.

We take system (6) as a nonlinear approximation of system (45). Below we will prove that the control $u=u(x)$, constructed in the previous section, solves the stabilization problem for system (45).

Let the control $u=u(x)$ defined by (7) solve the stabilization problem for system (6), and let the conditions of Theorem 2 hold. Let us show that this control solves the stabilization problem for system (45).

Assume that the control $u=u(x)$ is applied to system (45). Calculating the derivative of the Lyapunov function $V(x)$ given by (8) along the trajectories of closed-loop system (45), we obtain

$$
\left.\dot{V}(x)\right|_{(45)}=-(W x, x)+2 \sum_{i=s+1}^{n-1}\left(F h_{i+1}, x\right) x_{i}^{2 k_{i}+1}+2 \sum_{i=1}^{n-1}\left(F e_{i+1}, x\right) f_{i}(t, x, u),
$$

where $e_{i}$ is the $i$-th column of the $n \times n$ identity matrix.

Consider the case $n=2$. Then $s=0$. Thus we have

$$
\left.\dot{V}(x)\right|_{(45)}=-(W x, x)-2 b_{1}^{2} x_{1}^{2 k_{1}+2}+2\left(F e_{2}, x\right) f_{1}(t, x, u) .
$$


Using estimates (4), we deduce that

$$
\left.\dot{V}(x)\right|_{(45)} \leq-(W x, x)-2\left(b_{1}^{2}-\alpha_{1}\left\|F e_{2}\right\|\|x\|\right) x_{1}^{2 k_{1}+2}<0
$$

for $0<\|x\|<L_{1}, L_{1}=\min \left\{\rho, \frac{b_{1}^{2}}{\alpha_{1}\left\|F e_{2}\right\|}\right\}$.

Let us show that the last inequality holds. Indeed, if $x_{1} \neq 0$, then the inequality holds because the matrix $W$ is positive semi-definite and $b_{1}^{2}-\alpha_{1}\left\|F e_{2}\right\|\|x\|>0$ for $\|x\|<\frac{b_{1}^{2}}{\alpha_{1}\left\|F e_{2}\right\|}$. If $x_{1}=0$, then we have $-(W x, x)=-w_{22} \frac{a_{2}^{2}}{a_{1}^{2}} x_{2}^{2}<0$ for $x_{2} \neq 0$ and the inequality holds.

Now we consider the case $n \geq 3$. Using (43), from (46) we obtain

$$
\begin{aligned}
\left.\dot{V}(x)\right|_{(45)} \leq & -\left(\left(W-\lambda_{\min } I_{n, n-s}\right) x, x\right)-\sum_{i=1}^{s}\left(\lambda_{\min }-\sum_{j=s+1}^{n-1}\left|b_{i}^{j+1}\right|\left|x_{j}\right|^{r_{i}}\right) x_{i}^{2} \\
& -\sum_{i=s+1}^{n-1} g_{i}\left(x_{i}\right) x_{i}^{2 k_{i}+2}+2 \sum_{i=1}^{n-1}\left(F e_{i+1}, x\right) f_{i}(t, x, u)
\end{aligned}
$$

for $x \in \mathbb{R}^{n}$ such that $\left|x_{i}\right|<\min \left\{\beta_{i}, x_{i}^{*}\right\}, i=s+1, \ldots, n-1,\|x\| \neq 0$.

Let us choose the numbers $\varepsilon_{i}, i=1 \ldots n-1$ such that

$$
0<\varepsilon_{i}<\lambda_{\min }, \quad i=1, \ldots, s, \quad \text { and } \quad 0<\varepsilon_{i}<b_{i}^{i+1}, \quad i=s+1, \ldots, n-1 .
$$

Let $\widehat{x}_{i}$ be the smallest positive root of the equation $g_{i}\left(x_{i}\right)=\varepsilon_{i}, i=s+1, \ldots, n-1$. It is obvious that $\widehat{x}_{i}<x_{i}^{*}, i=s+1, \ldots, n-1$.

Then the following inequalities hold:

$$
\lambda_{\text {min }}-\sum_{j=s+1}^{n-1}\left|b_{i}^{j+1}\right|\left|x_{j}\right|^{r_{i}} \geq \varepsilon_{i}, \quad i=1, \ldots, s,
$$

for $\left|x_{j}\right| \leq \widehat{m}_{j}$, where

$$
\widehat{m}_{j}=\min _{1 \leq i \leq s}\left\{\left(\frac{\lambda_{\min }-\varepsilon_{i}}{(n-s-1)\left|b_{i}^{j+1}\right|}\right)^{\frac{1}{r_{i}}}\right\}, \quad j=s+1, \ldots, n-1,
$$

and

$$
g_{i}\left(x_{i}\right) \geq \varepsilon_{i}, \quad i=s+1, \ldots, n-1
$$

for $\left|x_{i}\right| \leq \widehat{x}_{i}, i=s+1, \ldots, n-1$.

Thus, from (48) and (49), it follows that inequality (47) takes the form

$$
\begin{aligned}
\left.\dot{V}(x)\right|_{(45)} \leq-((W & \left.\left.-\lambda_{\min } I_{n, n-s}\right) x, x\right)-\sum_{i=1}^{s} \varepsilon_{i} x_{i}^{2}-\sum_{i=s+1}^{n-1} \varepsilon_{i} x_{i}^{2 k_{i}+2} \\
+ & 2 \sum_{i=1}^{n-1}\left(F e_{i+1}, x\right) f_{i}(t, x, u)
\end{aligned}
$$


for $x \in \mathbb{R}^{n}$ such that $\left|x_{i}\right|<m_{i}$, where $m_{i}=\min \left\{\widehat{x}_{i}, \widehat{m}_{i}\right\}, i=s+1, \ldots n-1$. Using estimates (4), we have

$$
\sum_{i=1}^{n-1}\left(F e_{i+1}, x\right) f_{i}(t, x, u) \leq \sum_{i=1}^{n-1} \alpha_{i}\left\|F e_{i+1}\right\|\|x\|\|y\|^{2},
$$

where $y=\left(x_{1}^{k_{1}+1}, x_{2}^{k_{2}+1}, \ldots, x_{n-1}^{k_{n-1}+1}\right)^{T},\|y\|^{2}=\sum_{i=1}^{n-1} x_{i}^{2 k_{i}+2}$.

Using the notation

$$
\varepsilon=\min _{1 \leq i \leq n-1} \varepsilon_{i}, \quad m=\min _{s+1 \leq i \leq n-1} m_{i}
$$

from inequalities (50) and (51) we deduce that

$$
\left.\dot{V}(x)\right|_{(45)} \leq-\left(\left(W-\lambda_{\min } I_{n, n-s}\right) x, x\right)-\left(\varepsilon-\sum_{i=1}^{n-1} \alpha_{i}\left\|F e_{i+1}\right\|\|x\|\right)\|y\|^{2}<0
$$

for $0<\|x\|<L_{2}$, where $L_{2}=\min \left\{\rho, \frac{\varepsilon}{\sum_{i=1}^{n-1} \alpha_{i}\left\|F e_{i+1}\right\|}, m\right\}$.

Let us show that inequality (52) is true. To this end, we consider the following two cases. If $\|y\| \neq 0$, then $\varepsilon-\sum_{i=1}^{n-1} \alpha_{i}\left\|F e_{i+1}\right\|\|x\|>0$ for $\|x\|<L_{2}$. Hence, combining the last inequality with inequality (31), we obtain that inequality (52) is true. If $\|y\|=0$, then

$$
-\left(\left(W-\lambda_{\min } I_{n, n-s}\right) x, x\right)=-w_{s+1 s+1} \frac{a_{n}^{2}}{a_{s+1}^{2}} x_{n}^{2}<0 \quad \text { for } \quad x_{n} \neq 0
$$

and inequality (52) is true.

We have shown that the equilibrium point $x=0$ of the closed-loop system (45) is asymptotically stable, and thus we have proved that the control $u=u(x)$ defined by (7) solves the stabilization problem for system (45).

Let us construct an ellipsoidal approximation of the domain of attraction to the equilibrium point $x=0$ of the closed-loop system (45). To this end, we find $c>0$ such that the ellipsoid $(F x, x)<c$ is inscribed in the ball $\|x\| \leq L$, where $L=\left\{\begin{array}{l}L_{1}, n=2 \\ L_{2}, n \geq 3\end{array}\right.$. It is easy to verify that this ellipsoid has the form

$$
\Phi=\left\{x \in \mathbb{R}^{n}:(F x, x)<c, \quad c=\lambda_{\min }(F) L^{2}\right\},
$$

where $\lambda_{\min }(F)>0$ is the smallest eigenvalue of the matrix $F$. 
Now, basing on results obtained in this section, we state the following theorem.

Theorem 3. Suppose that the conditions of Theorem 2 hold. Then the control $u=u(x)$ defined by (7) solves the stabilization problem for system (45); and the domain of attraction to the equilibrium point $x=0$ of the closed-loop system contains ellipsoid (53).

Finally, we solve the stabilization problem for system (1) for the case where $c_{i}, i=1, \ldots, n-1$ are some real numbers such that $\prod_{i=1}^{n-1} c_{i} \neq 0$. So system (1) has the form

$$
\left\{\begin{array}{l}
\dot{x}_{1}=u \\
\dot{x}_{i}=c_{i-1} x_{i-1}^{2 k_{i-1}+1}+f_{i-1}(t, x, u), \quad i=2, \ldots, n .
\end{array}\right.
$$

We recall that the numbers $k_{i}$ satisfy condition (3) and the functions $f_{i}(t, x, u)$, $i=1, \ldots, n-1$ satisfy conditions (4).

The following theorem provides a way to solve the stabilization problem for nonlinear system (54), which is uncontrollable with respect to the first approximation.

Theorem 4. Suppose that the conditions of Theorem 2 hold. Put

$$
\widehat{L}_{1}=\min \left\{\frac{\rho}{\max _{1 \leq i \leq n} \widehat{c}_{i}}, \frac{b_{1}^{2}}{\widehat{\alpha}_{1}\left\|F e_{2}\right\|}\right\}, \quad \widehat{L}_{2}=\min \left\{\frac{\rho}{\max _{1 \leq i \leq n} \widehat{c}_{i}}, \frac{\varepsilon}{\sum_{i=1}^{n-1} \widehat{\alpha}_{i}\left\|F e_{i+1}\right\|}, m\right\} \text {, }
$$

where $\widehat{\alpha}_{i}=\frac{\alpha_{i}}{\left|\widehat{c}_{i+1}\right|} \max _{1 \leq j \leq n-1} \widehat{c}_{j}^{2 k_{j}+2}$ and $\widehat{c}_{i}$ are defined by the following relations

$$
\widehat{c}_{1}=1, \quad \widehat{c}_{2}=c_{1}, \quad \widehat{c}_{i}=c_{i-1}\left(\widehat{c}_{i-1}\right)^{2 k_{i-1}+1}, \quad i=3, \ldots, n .
$$

Then the control

$$
u(x)=a_{1} x_{1}+\frac{a_{2}}{\widehat{c}_{2}} x_{2}+\cdots+\frac{a_{n}}{\widehat{c}_{n}} x_{n}+\sum_{i=s+1}^{n-1} a_{n-s+i}\left(\frac{x_{i}}{\widehat{c}_{i}}\right)^{2 k_{i}+1}
$$

solves the stabilization problem for system (54). Moreover, the domain of attraction to the equilibrium point $x=0$ of the closed-loop system (54) contains the ellipsoid

$$
\Phi=\left\{x \in \mathbb{R}^{n}:\left(\widehat{C}^{-1} F \widehat{C}^{-1} x, x\right)<\lambda_{\min }(F) \widehat{L}^{2}\right\},
$$

where $\widehat{C}=\operatorname{diag}\left(\widehat{c}_{1}, \widehat{c}_{2}, \ldots, \widehat{c}_{n}\right)$ is a diagonal matrix of dimension $n \times n, \lambda_{\min }(F)>0$ is the smallest eigenvalue of the matrix $F$ and $\widehat{L}=\left\{\begin{array}{ll}\widehat{L}_{1}, & n=2 \\ \widehat{L}_{2}, & n \geq 3\end{array}\right.$. 
P r o o f. Suppose that the control $u=u(x)$ is applied to system (54). The change of variables $x=\widehat{C} y\left(x_{i}=\widehat{c}_{i} y_{i}, i=1, \ldots, n\right)$ maps system (54) to the system

$$
\left\{\begin{array}{l}
\dot{y}_{1}=v(y) \\
\dot{y}_{i}=y_{i-1}^{2 k_{i-1}+1}+\frac{1}{\widehat{c}_{i}} f_{i-1}(t, \widehat{C} y, u), \quad i=2, \ldots, n
\end{array}\right.
$$

where $v(y)=u(\widehat{C} y), y=\left(y_{1}, \ldots, y_{n}\right)^{T}$. From estimates (4) it follows that

$$
\left|\frac{1}{\widehat{c}_{i+1}} f_{i}(t, \widehat{C} y, u)\right| \leq \frac{\alpha_{i}}{\left|\widehat{c}_{i+1}\right|} \sum_{i=1}^{n-1}\left(\widehat{c}_{i} y_{i}\right)^{2 k_{i}+2} \leq \widehat{\alpha}_{i} \sum_{i=1}^{n-1} y_{i}^{2 k_{i}+2}, \quad i=1, \ldots, n-1
$$

for $\|y\| \leq \frac{\rho}{\max _{1 \leq i \leq n} \widehat{c}_{i}}$.

Thus, using Theorem 3, we obtain that the control

$$
v(y)=a_{1} y_{1}+a_{2} y_{2}+\cdots+a_{n} y_{n}+\sum_{i=s+1}^{n-1} a_{n-s+i} y_{i}^{2 k_{i}+1}
$$

solves the stabilization problem for the open-loop system (55). Moreover, the domain of attraction to the equilibrium point $x=0$ of the closed-loop system contains the ellipsoid

$$
\left\{y \in \mathbb{R}^{n}:(F y, y)<c, \quad c=\lambda_{\min }(F) \widehat{L}^{2}\right\} .
$$

Making the reverse change of variables $y=\widehat{C}^{-1} x$, we obtain the stabilizing control for system (54) and the ellipsoidal approximation of the domain of attraction to the equilibrium point $x=0$ of the closed-loop system.

E x a m p l e 1. Consider the system

$$
\left\{\begin{array}{l}
\dot{x}_{1}=u \\
\dot{x}_{2}=x_{1}-\frac{1}{2} \sin 2 x_{1} \\
\dot{x}_{3}=x_{2}-x_{2} \cos x_{2}-\sin x_{2}+\frac{1}{2} \sin 2 x_{2}
\end{array}\right.
$$

In this case

$$
\varphi_{1}(t, x, u)=x_{1}-\frac{1}{2} \sin 2 x_{1}, \quad \varphi_{2}(t, x, u)=x_{2}-x_{2} \cos x_{2}-\sin x_{2}+\frac{1}{2} \sin 2 x_{2} .
$$

Using the Taylor expansion formula, we obtain

$$
\varphi_{1}(t, x, u)=\frac{2}{3} x_{1}^{3}+f_{1}\left(x_{1}\right), \quad \varphi_{2}(t, x, u)=\frac{1}{12} x_{2}^{5}+f_{2}\left(x_{2}\right),
$$


where $\left|f_{1}\left(x_{1}\right)\right| \leq \frac{2}{15}\left|x_{1}\right|^{5}$ and $\left|f_{2}\left(x_{2}\right)\right| \leq \frac{1}{90}\left|x_{2}\right|^{7}$.

Thus system (56) takes the form of (54) with $n=3, k_{1}=1, k_{2}=2, c_{1}=\frac{2}{3}$, $c_{2}=\frac{1}{12}, \alpha_{1}=\frac{2}{15}, \alpha_{2}=\frac{1}{90}, \rho=1, s=0$. Then $\widehat{c}_{1}=1, \widehat{c}_{2}=\frac{2}{3}, \widehat{c}_{3}=\frac{8}{729}$.

Put $a_{1}=-1, a_{2}=-2, a_{3}=-\frac{1}{2}, w_{11}=2$. Then, according to (9) and (12), we have

$$
A=\left(\begin{array}{rrr}
-1 & -2 & -\frac{1}{2} \\
0 & 0 & 0 \\
0 & 0 & 0
\end{array}\right), \quad W=\left(\begin{array}{lll}
2 & 4 & 1 \\
4 & 8 & 2 \\
1 & 2 & \frac{1}{2}
\end{array}\right)
$$

From (17) and (25) we obtain that the solution of matrix equation (11) when $f_{22}=5, f_{33}=1$ has the form

$$
F=\left(\begin{array}{ccc}
1 & 2 & \frac{1}{2} \\
2 & 5 & \frac{5}{4} \\
\frac{1}{2} & \frac{5}{4} & 1
\end{array}\right)
$$

Thus, according to Theorem 4, we obtain that the control

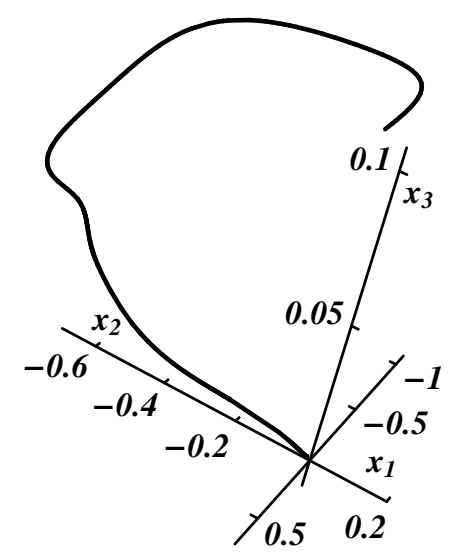

Fig. 1. Phase trajectory $x(t)$ in $\mathbb{R}^{3}$. 


$$
u(x)=-x_{1}-3 x_{2}-\frac{729}{16} x_{3}-\frac{5}{2} x_{1}^{3}-\frac{243}{16} x_{2}^{5}
$$

solves the stabilization problem for system (56); and the domain of attraction to the equilibrium point $x=0$ of the closed-loop system contains the ellipsoid

$$
\Phi=\left\{x \in \mathbb{R}^{3}:(F y, y) \leq 0.0891 \ldots\right\}, \quad \text { where } \quad y=\left(\frac{x_{1}}{\widehat{c}_{1}}, \frac{x_{2}}{\widehat{c}_{2}}, \frac{x_{3}}{\widehat{c}_{3}}\right)^{T} .
$$

Numerical analysis shows that we can take a point out of this ellipsoid. For example, let $x_{0}=(-0.1,-0.3,0.15)^{T}$ be the initial point. This point does not belong to the ellipsoid $\Phi$, but it belongs to the domain of attraction to the origin. Graphic of the phase trajectory is shown in Fig. 1.

\section{References}

[1] N.N. Krasovskii, Stabilization Problems for Controllable Motions. In: I.G. Malkin, Theory of Stability of Movement. Nauka, Moscow, 1966, p. 475-514.

[2] B.T. Polyak, P.S. Scherbakov, Robust Stability and Control. Nauka, Moscow, 2002.

[3] V.I. Korobov, The Controllability Function Method. R\&C Dynamics, MoscowIzhevsk, 2007. (Russian)

[4] N.K. Khalil, Nonlinear Systems. Prentice Hall, New York, 2002.

[5] M. Kawski, Stabilization of Nonlinear Systems in the Plane. - SCL (1989), No. 12, 169-175.

[6] V.I. Korobov and M.O. Bebiya, Stabilization of Some Class of Nonlinear Systems that are Uncontrollable in the First Approximation. - Dopov. Nats. Akad. Nauk Ukr., Mat. Pryr. Tekh. Nauky (2014), No. 2, 20-25. (Russian)

[7] M.O. Bebiya, Stabilization of Systems with Power Nonlinearity. - Visn. Khark. Univ., Ser. Mat. Prykl. Mat. Mekh. 69 (2014), No. 1120, 75-84. (Russian)

[8] V.I. Korobov and A.V. Lutsenko, Robust Stabilization of one Class of Nonlinear Systems. - Autom. Remote Control 75 (2014), No. 8, 1433-1444.

[9] W. Lin and C. Quan, Adding One Power Integrator: A Tool for Global Stabilization of High-Order Lower-Triangular Systems. - Systems Control Lett. 39 (2000), No. 5, 339-351.

[10] W. Lin and C. Quan, Adaptive Regulation of High-Order Lower-Triangular Systems: an Adding a Power Integrator Technique. - Systems Control Lett. 39 (2000), No. 5, $353-364$.

[11] Z. Sun and Y. Liu, State-Feedback Adaptive Stabilizing Control Design for a Class of High-Order Nonlinear Systems with Unknown Control Coefficients. - Jrl. Syst. Sci. \& Complexity 20 (2007), No. 3, 350-361. 
[12] G. Zhao and N. Duan, A Continuous State Feedback Controller Design for HighOrder Nonlinear Systems with Polynomial Growth Nonlinearities. - IJAC 10 (2013), No. 4, 267-274.

[13] V.I. Korobov, Controllability and Stability of Certain Nonlinear Systems. - Differentsial'nye Uravneniya 4 (1973), No. 4, 614-619. (Russian)

[14] G.M. Sklyar, K.V. Sklyar, and S.Yu. Ignatovich, On the Extension of the Korobov's Class of Linearizable Triangular Systems by Nonlinear Control Systems of the Class $C^{1}$. - Systems Control Lett. 54 (2005), No. 11, 1097-1108. 